\title{
Vacancy clusters, dislocations and brown colouration in diamond
}

\author{
U Bangert ${ }^{1}$, R Barnes ${ }^{1}$, M H Gass ${ }^{2}$, A L Bleloch ${ }^{2}$ and I S Godfrey ${ }^{1}$ \\ ${ }^{1}$ Nanostructured Materials Research Group, School of Materials, \\ The University of Manchester, PO Box 88, Manchester M1 7HS, UK \\ ${ }^{2}$ SuperSTEM, STFC Daresbury Laboratory, Warrington WA4 4AD, UK \\ E-mail: ursel.bangert@.manchester.ac.uk
}

Received 4 April 2009, in final form 25 June 2009

Published 19 August 2009

Online at stacks.iop.org/JPhysCM/21/364208

\begin{abstract}
Following on from the idea that clusters of vacancies are the origin of the featureless absorption and brown colouration in natural diamond, dislocations are shown to exhibit sub-bandgap absorption also. The vacancy cluster idea has arisen from theoretical predictions of $\pi$-bonded chains reconstructing the cluster surfaces and has been confirmed by energy loss studies. In contrast, bandgap states at dislocations are observed in brown and colourless diamonds alike, giving rise to weak absorption, which resembles that theoretically predicted from shuffle dislocation segments. This, however, would not account for the degrees of brownness in the diamonds, but it suggests that if such shuffle segments exist, vacancies must have been present and moved to dislocations to create these configurations in the first place. The question arises, what happens to the vast number of vacancy clusters upon high pressure high temperature (HPHT) annealing, which renders the diamonds colourless. Our observations on natural brown diamonds after HPHT treatment suggest that vacancy clusters, trapped in the strain fields of dislocations, grow in size accompanied by a decrease in their numbers; this leads to much reduced optical absorption.
\end{abstract}

(Some figures in this article are in colour only in the electronic version)

\section{Introduction}

The origin of the brown colour in type IIa natural and CVD diamond has been an active area of study for several years. Brown diamonds can be transformed into the colourless variety by HPHT treatment. To be able to tell whether such treatment has been applied, and, on a more fundamental level, to elucidate the origin of the colouration and the mechanism for its loss, is of considerable interest to gemmologists and gem traders. Other colourations, e.g., yellow, blue, red and grey can be attributed to point defects involving impurity atoms. However, brown type IIa diamond has few impurities and the brown colouration is largely due to native defects. It is believed that all brown natural diamonds have undergone plastic deformation at some stage in their past (although not all plastically deformed diamonds are brown), and since plastic deformation results in dislocations and there are high numbers of these in brown diamond, the question arises, in the light of lack of impurities, whether the colour is due to electronic states at dislocations.
Electronic states in the diamond band gap at dislocation cores have been found in brown diamond that are not seen as often in natural or HPHT treated colourless diamonds [1]. However, it is recognized that brown CVD diamonds can have very few dislocations, of density $10^{5} \mathrm{~cm}^{-2}$ compared with $10^{9} \mathrm{~cm}^{-2}$ in natural type IIa brown diamonds, and this implies that dislocations are not the sole source of the brown colouration. Fall et al [2] have carried out first principles calculations of energy levels at dislocations in diamond. They predicted that dislocation shuffle segments have $\mathrm{sp}^{2}$ bonded carbon core atoms, which create absorption peaks in the bandgap (from $2 \mathrm{eV}$ upwards), and hence could make the dislocation optically/electrically active. The absorption would be largely featureless, much like that of brown diamond. We can estimate the required number of core atoms to produce an absorption comparable to that seen experimentally. The absorption at $2.5 \mathrm{eV}$ due to the 438 -atom cell containing 2 core atoms of a shuffle dislocation is $5030 \mathrm{~cm}^{-1}$. Hence, for $0.1 \mathrm{~cm}^{-1}$ absorption we require $2 \times 10^{16}$ core atoms $\mathrm{cm}^{-3}$ or a shuffle dislocation density of $6 \times 10^{8} \mathrm{~cm}^{-2}$. Estimates of 
the dislocation density found in single crystal natural type IIa brown diamonds by transmission electron microscopy (TEM) studies [3] are about $10^{9} \mathrm{~cm}^{-2}$ both before and after the HPHT treatment to remove the colouration. Thus we require a significant fraction of the dislocations in brown diamonds to be in the shuffle form, which seems unlikely, as the stability for the $90^{\circ}$ glide dislocation is much larger then for shuffle dislocations. Transformation of the dislocation cores upon heat treatment would tend to eliminate these active metastable centres and transform the shuffle into inactive glide segments.

Previous experimental studies have correlated extended defects in undoped CVD diamond [4, 5] and natural type II diamond [6, 7] with band-A cathodoluminescence at 2.8$2.9 \mathrm{eV}$, which in turn has been associated with carbon atoms in reduced coordination [5], and recent electron energy loss spectroscopy (EELS) studies of the absorption edge onset suggest that $\mathrm{sp}^{2}$-bonding is linked with dislocation cores [8] in natural brown diamond. Although it has not yet been possible to prove the existence and determine the density of shuffle segments in diamond, it is highly unlikely that every dislocation is a shuffle dislocation along its entire length. Besides, no conclusive evidence has been found of a difference in dislocation arrangements and numbers between brown and colourless diamond [3]. The above findings, and the absence of any other structural defects, lead to the suggestion that the brown colour might be due to something different altogether, namely point defect aggregates. Positron annihilation has revealed vacancy related defects in brown, natural type IIa diamond, showing up through characteristic lifetimes of 132 and 434 ps, for the monovacancy and vacancy clusters of at least 30 vacancies, respectively $[9,10]$. The vacancy cluster concentration was found to decrease in high temperature anneals; a loss in the brown colour was also observed. Enhanced positron trapping under illumination was found to correlate with optical absorption, suggesting that the optical transitions of vacancy clusters could be responsible for the brown colouration [10]. EELS showed enhanced absorption in the loss regime corresponding to $\pi$-transitions. On the basis of ab initio calculations Hounsome et al predict that the surfaces of vacancy clusters or chains in diamond will be lined with $\pi$-bonds [11]. Vacancy 'disks' or 'chains' lying in the $\{111\}$ planes will exhibit featureless optical absorption spectra similar to brown diamond [11]. Theory further suggests that the vacancy clusters could collapse into prismatic dislocation loops with HPHT treatment [12]. Positron annihilation experiments [13,9] on a variety of type IIa diamonds demonstrate that the concentration of these 'large' 30-60 vacancy clusters is greatest in brown CVD and natural brown diamond, reducing with HPHT treatment and is smallest in natural colourless diamonds. How these vacancies are introduced in the first place is not clear, however, it appears that in any case their distributions might be intimately linked with dislocations and their motion.

Subsequent to these findings, an investigation into vacancy cluster visibility in high-resolution transmission electron microscopy (HR-TEM) has shown nm-scale contrast $[14,15]$. Transitions involving $\pi$-bonds in carbon are readily detectable in EELS. Therefore, spatially resolved EEL studies have been carried out, in which spectrum images [16] were acquired and compared in dislocation-free regions of the brown and colourless zones of a diamond containing brown and colourless banding, here upon termed a 'zebra' diamond [17]. Results shown in figures 1(c) and (d) are HREM images obtained from zones in the zebra diamond. Figures 1(e) and (f) clearly demonstrate an increased intensity in the energy region associated with a $\pi$-electron system in the brown zone [17]. The images in figures 1(b) and (d) furthermore display the contrast expected of high densities of nm-size cavities within a crystal structure $[15,19]$.

In this paper we re-visit the still unresolved issues concerning the possible role of dislocations in the brownness, and also the fate of the vacancy clusters after annealing.

\section{Methodology}

Natural brown, colourless and HPHT annealed natural brown diamonds were laser cut and subsequently ion beam milled to electron transparency. For the study of subtle effects, e.g., at dislocation cores, the Daresbury SuperSTEM with an aberration corrected probe was used: atomic resolution HAADF and BF images with the dislocation line parallel to the e-beam aid in locating the core precisely. Highly spatially resolved EELS was carried out in spectrum imaging mode [16].

Post-HPHT-treatment studies to assess the destiny of the vacancy clusters over larger areas were carried out in the NorthWest STEM; this instrument does not have aberration correction but an equivalent EELS facility.

EELS was carried out at high energy dispersion to access the low loss regime $2-10 \mathrm{eV}$ with the highest possible energy separation of the low loss features. The instrumental resolution is $\sim 0.3 \mathrm{eV}$. EEL spectrum images (SIs) [16] were acquired with raster sizes varying from 10-200 nm in length and pixel sizes ranging from $0.3-4 \mathrm{~nm}$. These SIs were analysed for the occurrence of $\mathrm{sp}^{2}$ bonding and the spatial correlation of these bonds with microstructural features. $\mathrm{Sp}^{2}$ bonding can be detected by the enhancement of the energy loss signal in the 4$7 \mathrm{eV}$ regime, and the spatial distribution visualized by mapping the intensity of the energy loss signal in the particular energy region in spatial relation to the microstructural image. The energy loss intensity in the entire energy regime is influenced by diffraction contrast and thickness [18]; the former becomes manifest in a linear variation of the ZLP intensity (and also roughly of the entire spectrum intensity), the latter in relative plasmon intensity changes and multiple scattering peaks across the SI. A correction script was developed and applied to all SIs shown in the following. This reduces the entire SI to a single scattering distribution, followed by normalization of thickness and diffraction effects via division of the SI by maps of the thickness and the total intensity.

\section{Results}

\subsection{Natural brown diamond}

Images 1 (a) and (c) show colourless regions in a type IIa brown diamond after HPHT treatment and in a colourless band 

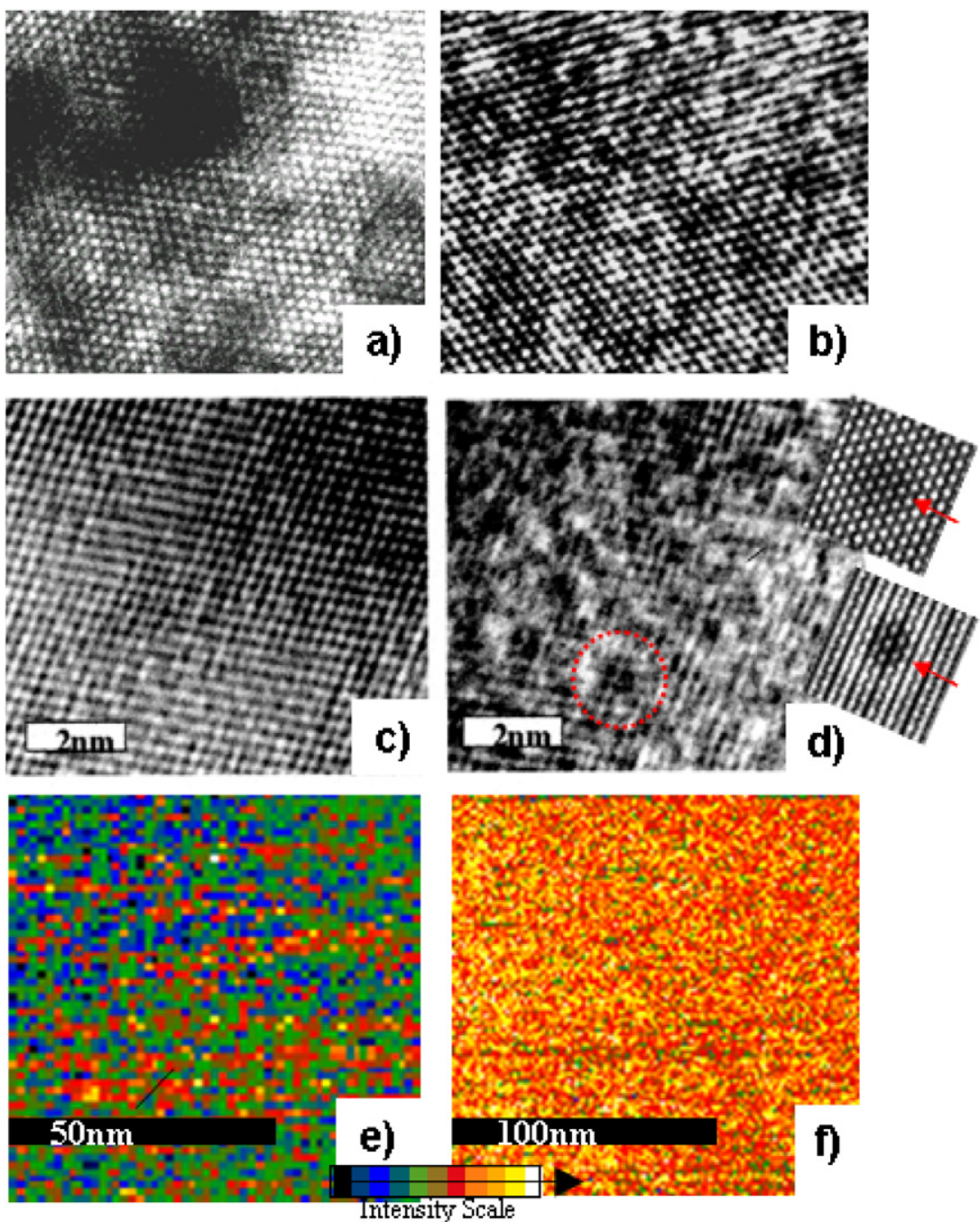

Figure 1. Experimental HR-TEM images (a) of HPHT treated and (b) of untreated brown diamond, (c) of a colourless and (d) of a brown zone in a 'zebra' diamond, all at $-150 \mathrm{~nm}$ defocus. Alongside (d) are shown TEMSIM simulations at $-150 \mathrm{~nm}$ defocus, for a vacancy cluster (52 vacancies in a 650-atom supercell, $\langle 110\rangle$ projection) in diamond, placed at the entrance surface (top) and the middle (bottom) of an $80 \mathrm{~nm}$ thick sample. All parameters used ( $300 \mathrm{kV}$ accelerating voltage, $40 \mathrm{mrad}$ objective aperture) are as in the experiment. Beam coherence was assumed in the simulations and a cut-off filter for frequencies higher than 200 reflections were applied to the Fourier transform of the simulated images, before the inverse transform (shown here) was performed. (e), (f) Maps of the relative EEL intensity at 4-6 eV, taken away from dislocations in a colourless and a brown zone in the zebra sample in (c) and (d). The intensity is on a temperature scale (i.e., blue: low, yellow: high).

of the zebra diamond, respectively. Images $1(\mathrm{~b})$ and $(\mathrm{d})$ show regions of optically similar degrees of brownness in a brown type IIa diamond and in a brown band of the zebra diamond. The speckle contrast in figures 1 (b) and (d) agrees well with HREM simulations of vacancy clusters at high defocus. The high defocus is important for showing up the clusters by their Fresnel contrast, superimposed on the lattice image [14]. We have attempted to quantify the 'speckle contrast' in HREM images [19]. To use computational image recognition methods in order to identify and count vacancy clusters would require immensely complicated software, without any guarantee of reliable results. Hence 'manual' counting based on visual recognition was deemed to be the most appropriate method, even though it can introduce some subjective judgement. Although there are contrast variations in type II colourless and HPHT treated, originally brown diamond (presumably evoked by rough and corrugated surfaces, see figure 1(a)), brown diamond shows the small-speckle contrast to a much greater extent. The amount of contrast that can be attributed to bulk defects rather than surface roughness has been determined by comparing thick and thin regions of the same area; this method consistently shows an increase in the number of clusters in brown over that in colourless diamond, giving a concentration $10^{17} \mathrm{~cm}^{-3}$, which is physically reasonable and correlates with predictions by positron annihilation, of around $10^{16} \mathrm{~cm}^{-3}$ clusters containing 50-60 vacancies, but also of an unspecified concentration of clusters of around 20 vacancies [13]. Evidence of clusters of $\pi$-bonds in core loss EELS, and the concentrations deduced from HR-TEM thus appear to correlate with positron annihilation.

High densities of vacancy clusters should cause a strong presence of $\mathrm{sp}^{2}$-bonds, which can be conveniently imaged in 
EELS. The localization of scattering with low energy losses is of the order of a few nm; given the small cluster spacing at the above densities, $\mathrm{sp}^{2}$ bonding would thus appear as almost omni-present in SIs with nm-pixel sizes. If the vacancy cluster hypothesis was true then this $\mathrm{sp}^{2}$-related intensity should furthermore be independent of dislocations. The intensity maps in figures 1(e) and (f) of energy losses at 4-6 eV, obtained from brown and colourless regions of the same 'zebra' diamond as in figures $1(\mathrm{c})$ and (d) prove this. The zebra diamond is particularly suited for this kind of study as it excludes effects of variations in the STEM and EELS systems over several days and also variations that would occur from sample to sample (e.g., due to sample preparation). The EEL intensities are displayed on the same scale, and are strongly enhanced in the brown region.

\subsection{Dislocations}

The improvements with aberration corrected imaging have enabled dislocation cores to be pinpointed for EELS with Angstrom spatial resolution. The dislocation in figure 2(a) consists of a (111) half-plane (red lines mark lattice planes to guide the eye) and is likely to be of the mixed $60^{\circ}$ type. These dislocations dominate in natural diamond. The dislocation in figure 2(a) is shown here to be electronically active, as additional sub-bandgap states are seen at its core. This can be seen from the EEL intensity map of energy losses at 4-7 eV in figure 2(b). In order to extract the EEL intensity distribution the EEL spectrum image was corrected for sample thickness and diffraction contrast effects, as stated in section 2. Whether the dislocation belongs to the optically active shuffle dislocation group, however, can still not be resolved in the HREM images. According to the theoretical model the shuffle dislocation possesses more states deeper in the gap (figure 2(d)) than the glide partial or the screw dislocation (figures 2(e), and (f)), and the experimental difference spectrum in figure 2(c), extracted from spectra of the dislocation core region and away from the core in figure 2(a), resembles more that of the shuffle in figure 2(d). The calculations show EEL spectra for a variety of $E$-field directions of the passing electron. In reality the $E$-field vectors of the electron are distributed with their tips marking the surface of an ellipsoid with the long axis parallel to the direction of the electron, hence the observed EEL spectrum is a superposition of all the theoretical spectra, exhibiting peaks at $\sim 3.5$ and $\sim 6 \mathrm{eV}$, which is in remarkably good agreement with figure 2(d) with peaks at 4 and $6 \mathrm{eV}$. Note the high spatial resolution of the EELS information of $\sim 2 \mathrm{~nm}$ that has been achieved here in the low loss regime. Interestingly this dislocation is of a natural, colourless sample. Similar results have been obtained from several dislocations in colourless and brown samples. Due to the highly specialized nature of experiment and equipment (only very few dislocations like this can be found and have their data taken in one session) and the limited time allocation on the instruments, building up meaningful statistics from results like this would take months, if not years, of sessions.
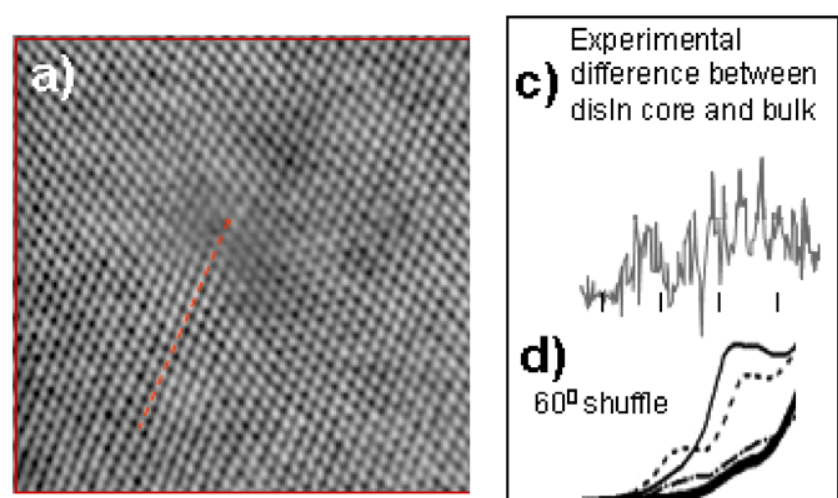

c) difference between
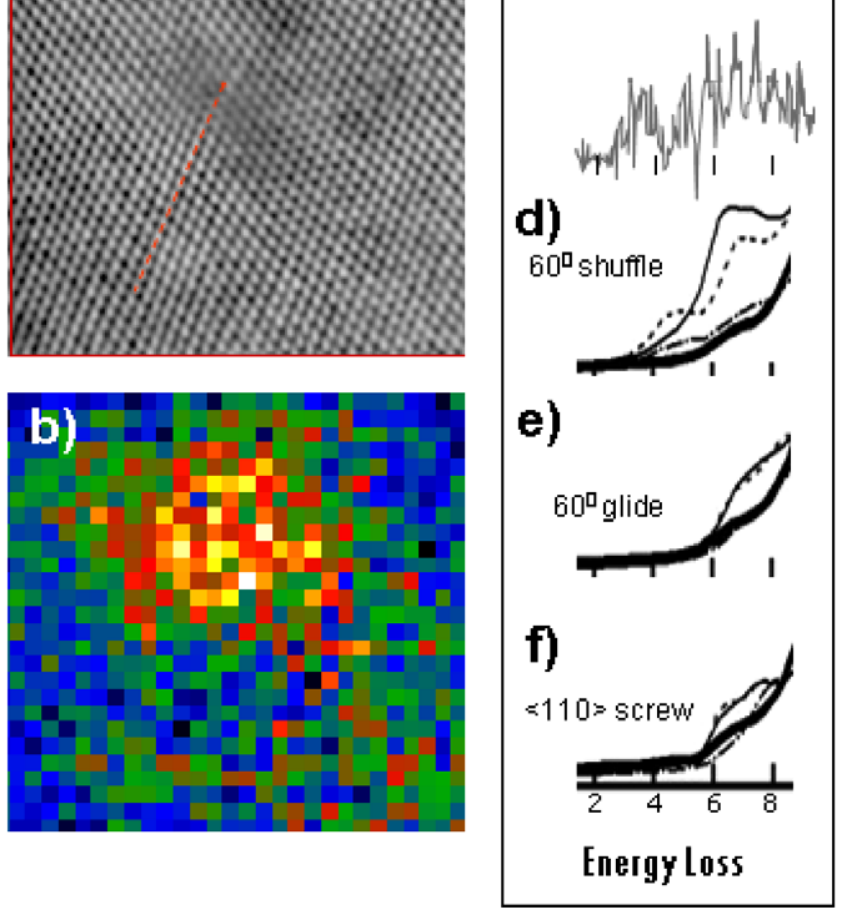

Figure 2. (a) HAADF STEM image of the diamond lattice of a colourless diamond, noise removed by Fourier filtering, showing a dislocation by the ending (111) plane, marked in red, with the dislocation core aligned parallel to the electron beam. The frame width of the image is $7.5 \mathrm{~nm}$. (b) Map of the EEL intensity at 5.4-7.4 eV created from a thickness and diffraction corrected and normalized SI of the region in (a). The intensity is on a similar scale as in figure 1. (c) Difference between spectra extracted from the dislocation core region (yellow/orange pixels) and from a region remote from the core (e.g., blue pixels in the bottom left corner of (b)). (d) Low loss EEL spectrum modelled using ab initio calculations [2], of a shuffle partial dislocation in diamond, (e) same of a glide partial and (f) of a screw dislocation. The theoretical spectra are for $E$-field directions $\langle 112\rangle$ perpendicular to the dislocation line (solid lines), $\langle 111\rangle$ perpendicular to the dislocation line (dashed lines), $\langle 110\rangle$ parallel to the dislocation line (dash-dotted lines) and for bulk diamond (thick solid line).

\subsection{High temperature high pressure treated natural brown diamond}

If the dislocation in figure 2 was a shuffle dislocation and representative of the dislocations in this sample, one might wonder whether brown colouration could have been removed when vacancy clusters were dissolved and some of the vacancies migrated to dislocation cores, transforming glide into shuffle cores, following, e.g., some event in the geological history of the sample. But even if brownness in diamonds could be made to disappear in this way, the sheer number of vacancies occurring in brown samples would still not be reduced sufficiently by the dislocation cores (estimates give $5 \times 10^{14}$ vacancies $\mathrm{cm}^{-2}$ in brown diamond, on the assumption of 50 vacancies per cluster in a $100 \mathrm{~nm}$ thick sample, compared to $5 \times 10^{11}$ vacancies that can be accommodated by $10^{9}$ shuffle dislocations $\mathrm{cm}^{-2}$ in annealed diamond in a $100 \mathrm{~nm}$ 
thick sample), and the question remains, where the rest of the vacancies go. We have carried out EELS investigations, similar to those shown in figure 1, of larger areas in HPHT diamonds to find out whether there was a comparable high background of $\mathrm{sp}^{2}$ related EELS intensity in these annealed samples. If $\mathrm{sp}^{2}$ was related to the brown colour, then after HPHT treatment, when the diamonds have lost most of the brownness, the $\mathrm{sp}^{2}$ content should have significantly reduced, or a drastic re-distribution and re-arrangement of the centres responsible should have taken place.

Applying the same evaluation procedures as in the previous cases, EEL intensity maps reveal singular patches of enhanced $\mathrm{sp}^{2}$-related intensity with a spatial extent of 10$15 \mathrm{~nm}$ in diameter in the vicinity of many dislocations. The example in figure 3 shows that such EEL intensity patches do not correspond to contrast features in the BF images, and hence are not directly related to microstructural features, such as e.g., graphitic platelets (as have been observed in dark brown diamonds [20]), however, the constituting defect aggregates might be trapped in the strain field of dislocations. From the considerable number of SIs we have taken of HPHT diamonds we are not aware that such features exist remote from dislocations, but to be entirely sure EEL mapping of extensive areas would be required.

Assuming these defects are larger vacancy clusters and the optical absorption is proportional to the density of vacancy clusters times their surface area, we can then estimate whether the increase in cluster size and decrease in density could lead to loss of brownness: making an approximation based on observations in figures 1 (b) and (d) we consider the clusters in the natural brown diamond to be below $1 \mathrm{~nm}$ in diameter, and their density to be $10^{13} \mathrm{~cm}^{-2}$. The EEL intensity features in figure 3, from which we infer the existence of clusters, appear to be of the order of some ten nm in diameter, but the spatial extend of the low loss EELS signal does not give accurate sizes due to the delocalization of low energy inelastic scattering events; it is likely that the clusters here are between 10 and $20 \mathrm{~nm}$ in diameter. This would lead to a $\sim 10^{3}-$ $10^{4}$ times increase in the cluster volume from that in brown diamond and hence to an at least $10^{2}$ fold increase in the surface area of each cluster. Based on observation of $\sim 1-$ 2 clusters along each dislocation line segment, the estimated density of which is $10^{9} \mathrm{~cm}^{-2}$ [3], the ratio in the optical absorption in brown and annealed diamond is then at least $100: 1$. So it is possible that the observed reduction in the cluster density and an increase in their size significantly reduce the optical absorption and hence the brownness. Vacancy clusters of these large sizes would remain undetected in positron annihilation experiments. The question of course arises, how such large vacancy clusters can be stable, and what constellation of the vacancies is required. It has been suggested by theory [12] that vacancies aggregate in disks or octahedral voids, and experimental values of absorption coefficients in brown diamond consolidate calculated numbers of $\sim 100$ vacancies per aggregate. In the HPHT case in figure 3 the aggregates would contain significantly more (two orders of magnitude) vacancies, and TEM evidence suggests they might be in the shape of large platelets [21].
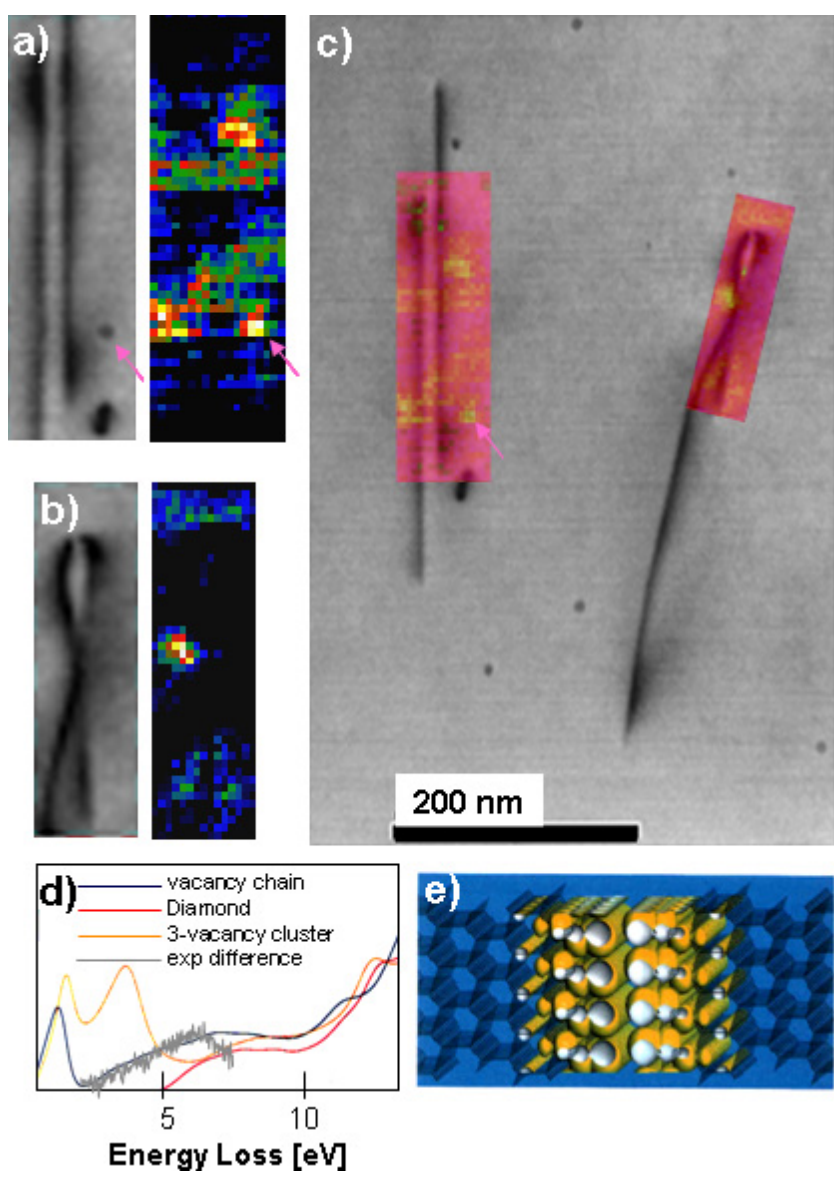

Figure 3. (a) and (b) Dislocation dipoles, in a HTHP treated diamond, imaged in STEM bright field in each left-hand panel, and $\mathrm{sp}^{2}$ related EELS intensity maps (4.6-5.4 eV; with EELS SI analysis and colour scale as described before) in each right-hand panel. Note the patches of high $\mathrm{sp}^{2}$ intensity (yellow), $10-15 \mathrm{~nm}$ in size. The intensity patch in (a) marked with an arrow, is due to a copper particle; all dark circular spots are Cu-particle contamination originating from the support grid. (c) Lower magnification overview with EEL intensity maps overlaid. The colour coding is different from (a) and (b) to provide transparency for the diffraction contrast of the dislocations; here pink colours signify low, and yellow colours enhanced intensity at 4.6-5.4 eV. (d) CASTEP simulations of the low loss EELS spectra of pure diamond (pink), a 3-vacancy cluster in a 64-atom cell (yellow), a (111) chain of vacancies (blue). An experimental difference spectrum (grey) of the bright spot and a black region in (b) is overlaid. (e) 3D visualization of the (111) vacancy chains. The bonds, atoms and/or unit cells are represented in dark grey. The orbitals of any states in the diamond bandgap are featured in yellow.

EEL spectra of vacancy defects, a (111) $\pi$-bonded vacancy chain and a 3-vacancy structure, were simulated with the CASTEP program and the Materials Studio package. The bonds in the 3-vacancy cluster are different from those in $\pi$-bonded chains; the latter are lining the inside of larger vacancy clusters. The low loss intensity of the $\pi$-bonded chain (figure 3(d), dark blue curve) is in much better agreement with the experimental observations (figure 3(d), grey curve, showing the difference in low loss signal at the location of the bright spot and the black area below in figure 3(b)) than that of the 3-vacancy cluster (figure 3(d), yellow curve). This 
does not mean that vacancy chains are the defect causing the brown colour, but that it is $\pi$-bonded carbon that is involved in the defect. The orbitals for a $\pi$-bonded vacancy chain are visualized in figure 3(e); the orbitals in the diamond bandgap are featured in yellow and light grey on the dark grey crystal lattice. The representation shows that states extend far into the vacancy but diminish quickly into the crystal.

In conclusion, further to investigations into the brown colouration in type IIA diamonds as being due to small vacancy clusters, we here investigate the fate of these vacancy clusters. We give evidence, via EELS, of the existence of bandgap states at dislocations in natural diamond- brown and colourless. These states give rise to EEL spectra resembling those of shuffle dislocation cores. The states are too small in number to cause significant optical absorption, however, formation of shuffle cores involves climb, suggesting presence of vacancies; these might have been created upon dissolution/re-formation of small vacancy clusters. We have also observed features that suggest the existence of large vacancy clusters in diamonds which have lost their brown colour upon HPHT treatment. This again suggests dissolution/re-formation or growth of small clusters into larger ones, which are optically inactive.

\section{References}

[1] Kolodzie A T 2001 PhD Thesis Cambridge

[2] Fall C J, Jones R, Briddon P R, Blumenau A T, Frauenheim T, Gutiérrez-Sosa A and Bangert U 2002 Phys. Rev. B 65205206

[3] Willems B 2005 Presented at 56th Diamond Conf. (Oxford, July)
[4] Ruan J, Kobashi K and Choyke W J 1992 Appl. Phys. Lett 603138

[5] Takeuchi D, Watanabe H, Yamanaka S, Okushi H, Sawada H, Ichinose H, Sekiguchi T and Kajimura K 2001 Phys. Rev. B 63245328

[6] Hanley P L, Kiawi I and Lang A R 1977 Phil. Trans. R. Soc. A 284329

[7] Kiawi I and Lang A R 1974 Phil. Mag. 30219

[8] Kolodzie A T and Bleloch A L 2003 Inst. Phys. Conf. Ser. 179 319

[9] Avalos V and Dannefaer S 2003 Physica B 340-342 76

[10] Saarinen K 2005 Presented at 56th Diamond Conf. (Oxford, July)

[11] Hounsome L, Jones R, Martineau P, Shaw M, Briddon P, Öberg S, Blumenau A T and Fujita N 2005 Phys. Status Solidi a 2022182

[12] Hounsome L et al 2006 presented at SBDD XI

[13] Mäki J M, Ranki V, Saarinen K, Martineau P and Fisher D 2004 presented at SBDD X

[14] Barnes R, Bangert U and Martineau P 2006 Phys. Status Solidi a 2033081

[15] Bangert U, Barnes R, Hounsome L S, Jones R, Blumenau A T, Briddon P R, Shaw M J and Öberg S 2006 Phil. Mag. 864757

[16] Hunt J A and Williams D B 1991 Ultramicroscopy 3847

[17] Barnes R, Bangert U and Willems B 2009 Diamond Relat. Mater. submitted

[18] Bangert U, Harvey A J, Jones R, Fall C J, Blumenau A T, Briddon R, Schreck M and Hoermann F 2004 New J. Phys. 6184

[19] Barnes R 2007 PhD Thesis Manchester

[20] Barnes R, Bangert U, Martineau P, Fisher D, Jones R and Hounsome L 2005 Inst. Phys. Conf. Ser. 26157

[21] Godfrey I S, private communication 